\title{
TECHNOLOGY OF CULINARY (FRYING) FATS
}

\author{
Oleksiy Udovenko ${ }^{1}$ \\ aleksey.udovenko@gmail.com \\ Fedir Gladkiy \\ gladky2009@gmail.com \\ Ivan Shkredov \\ john.shkredov@gmail.com \\ Kateryna Havriushenko ${ }^{1}$ \\ katealefarova@gmail.com \\ Olena Litvinenko ${ }^{1}$ \\ elena.litvinenko14@gmail.com \\ Katerina Kunitsia \\ Department of Innovative Food and Restaurant Technologies \\ Kharkiv Trade and Economic Institute of Kyiv National Trade and Economic University \\ 8 O. Yarosh lane, Kharkiv, Ukraine, 61045 \\ ekaterina.kunitsia@gmail.com \\ ${ }^{1}$ Department of Technology of Fats and Fermentation Products \\ National Technical University «Kharkiv Polytechnic Institute» \\ 2 Kyrpychova str., Kharkiv, Ukraine, 61002
}

\begin{abstract}
The increased popularity of deep-fried foods has put health and safety concerns on the agenda. Factors that affect the quality and safety of culinary (frying) fats include the fatty acid profile, as well as cooking modes and time, especially when renewal rates are low. The industry is currently facing a top-of-mind technology challenge: to reduce the "oil content" of fried foods by developing a frying fat that would meet up-to-date food production standards. In other words, there is a need for a frying fat, which should last as long as possible without oxidizing and changing colour. Furthermore, its uptake by foods, fried in it, must be $40-50 \%$ less than that of conventional fats. To this end, the authors put forward an innovative technology for producing culinary (frying) fats through esterification of fatty acids with high molecular weight alcohols. The research work that has been carried out so far prove the possibility of producing specialty fats by such modification to the applicable regulatory requirements: the melting range is between 29.6 and $34.65^{\circ} \mathrm{C}$, the acid value is up to $0.4 \mathrm{mg} \mathrm{KOH} / \mathrm{g}$. The viscosity of modified fats is lower than that of oils, which is due to the fat structure, and this circumstance will have a positive effect on the fat content in a finished product. The researchers has identified a numerical relationship (expressed as a regression model) of the yield of the desired fraction of a modified fat versus the ratio of reagents, reaction time, and process temperature, as well as calculated rational process parameters.

The aim of the research is to develop the technology of obtaining culinary (frying) fats by esterification of fatty acids with high molecular weight alcohols.
\end{abstract}

Keywords: frying fat, esterification, acid value, oxidation, melting point, modified fat.

DOI: $10.21303 / 2504-5695.2020 .001318$

\section{Introduction}

The existing market economy gives rise to significant developments in nutrition patterns. The fast pace of our life forces us to save time, including meal time, which undoubtedly means popularity of fast food, where the frying technology and fried foods dominate. In the United States, the market for commercial use of frying fats is estimated at 83 billion US dollars and is at least twice the size of the rest of the world [1-4]. Fried foods have pleasing savour, colour and texture, which make them very popular with consumers. The main requirements for frying fat: light color and transparency, no foam on the surface and smoke, high oxidative stability $[5,6]$. However, the existing deep frying technology causes fast formation of fat oxidation and polymerization products 
that are harmful for the body and inhibit digestion of finished foods. Therefore, the quality of frying fats and finished products is reduced. To this end, the development of new culinary (frying) fats and methods of preserving their quality becomes important.

The purpose of creating an "ideal" frying fat is to minimise processes of thermal deterioration. At a frying temperature of $160-180{ }^{\circ} \mathrm{C}$ oil undergoes a number of processes that affect the product quality. Chemical fat modifications, caused by frying, include hydrolysis, oxidation, oxidative polymerization, thermal polymerization, etc. When oil is exposed to thermal treatment for a long time, the weight content of free fatty acids grows, which is indicative of the product quality [9]. There is a change in the organoleptic properties of fat, it darkens, acquires a bitter taste and an unpleasant odor. The content of fat-soluble vitamins and polyunsaturated fatty acids in fat decreases, substances that are not absorbed by the body are formed. Pigments, contained in fats, carotenoids, chlorophyll and their derivatives are quite easily destroyed by thermal oxidation. During long frying, frying fat is subjected to further oxidation of hydrolysis products - to the formation of primary oxidation products - hydroperoxides, peroxides, epoxides, as well as secondary oxidation products - carbonyl and polymeric compounds. As a result of long-term use of frying fats, their pyrolysis occurs, cyclic compounds and toxic acrolein are formed [10].

With these processes in mind, a hypothesis was formulated on improving the technology of fat modification through esterification of fatty acids with high molecular weight alcohols. This hypothesis is confirmed by the use of blubber in cooking, which had been practiced before a moratorium was adopted on commercial whaling by the International Whaling Commission (IWC). Specifically, whale blubber contains esters of high molecular weight alcohols and high molecular weight fatty acids [11].

The proposed new technology, in particular, esterification of fatty acids with high molecular weight alcohols as frying fats, helps address the issue of thermal fat deterioration. Esters of fatty acids and high molecular weight alcohols are poorly hydrolysable, and, therefore, their use as culinary (frying) fats has a high potential. Use of esters of fatty acids and high molecular weight alcohols as frying fats is a promising area, which enables removal of adverse processes during frying.

\section{Materials and Methods}

The following reagents and materials were used in this study:

1. Stearic acid by MH \& Gustav Heess Ukraine.

2. Oleic acid by $\mathrm{MH} \&$ Gustav Heess Ukraine.

3. Cetostearyl alcohol (50:50, 30:70) by MH \& Gustav Heess Ukraine.

4. Cetyl alcohol by MH \& Gustav Heess Ukraine.

For esterification of fatty acids, we selected Lipozyme 435, a an immobilised lipase for biocatalysis on a silica gel granulated carrier by Novozyme (Denmark), which ensures synthesis in a mild environment, without secondary processes.

The products of esterification are esters of fatty acids of high molecular weight alcohols and water. This reaction is reverse, therefore, increasing the yield of esters requires shifting the equilibrium of the reaction towards the products [8]. The equilibrium can be shifted by withdrawing water from the reaction medium. The equation of the reaction is shown in Fig. 1.

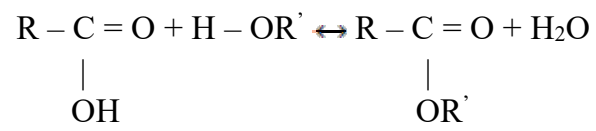

Fig. 1. Esterification reaction

To remove water from the reaction volume, NaA and KA type synthetic zeolites by SORBISGROUP (Russia) were used. A characteristic property of zeolites is the absorption of a substance that occurs through channels or window entrances that have certain dimensions. Only molecules, smaller than the channel diameter, can penetrate the zeolite through these channels. This makes it possible to use zeolites as molecular sieves [12]. The openings at the entrance to the pores are accurately fitted and for synthetic NaA zeolites they correspond to molecular sieves $4 \AA$, for KA - $3 \AA$. 
To calculate the rate of conversion of fatty acids to corresponding esters, for each reaction an acid value (AV) is determined as per ISO 660:1996, NEQ.

The acid value is used as the criterion, by which the reaction yield is measured. The acid value for cooking oils and fats should not exceed $0.4 \mathrm{mg} \mathrm{KOH} / \mathrm{g}$, therefore the samples were neutralized with a $10 \%$ water/alcohol/glycerin solution of $\mathrm{KOH}$. During the process of synthesis, the acid value was determined and the product yield was calculated.

The modified fat was examined in three repetitions. At the specified probability $\mathrm{P}=95 \%$, relative error in the acid value measurement did not exceed $2 \%$.

\section{Experimental procedures}

The reaction of esterification of fatty acids and high molecular weight alcohols resulted in four samples: cetyl ester of oleic acid, cetyl ester of stearic acid, cetostearyl ester of stearic acid, and cetostearyl ester of oleic acid. The results obtained are shown in Table $\mathbf{1}$.

Table 1

Acid values of esters of fatty acids of high molecular weight alcohols and their yields

\begin{tabular}{ccc}
\hline Item No. & Ester & Yield, $\%$ \\
\hline 1 & Cetyl ester of stearic acid & 98.64 \\
2 & Cetyl ester of oleic acid & 97.87 \\
3 & Cetostearyl ester of stearic acid & 98.26 \\
4 & Cetostearyl ester of oleic acid & 99.72
\end{tabular}

The yield of cetostearyl ester of oleic acid is the highest (99.72\%), therefore it was decided to fulfil the experiment plan, based on this sample.

The search of rational conditions for the esterification of fatty acids and high molecular weight alcohols is performed with the use of a full first-order factorial experiment. The plan of the experiment for the esterification of fatty acids and high molecular weight alcohols, which contains all possible combinations of both natural and coded factors, is presented as a planning matrix. For the planning of the experiments and processing of the experimental data, we used mathematical methods, as well as Statistica and Microsoft Excel software. The planning matrix and experimental results are shown in Table 2.

Table 2

Planning matrix and experimental results

\begin{tabular}{|c|c|c|c|c|c|c|c|c|}
\hline \multirow{2}{*}{$\begin{array}{c}\text { Experiment } \\
\text { No. }\end{array}$} & \multicolumn{3}{|c|}{ Natural factors } & \multirow{2}{*}{$X_{0}$} & \multicolumn{3}{|c|}{ Coded factors } & \multirow{2}{*}{$\begin{array}{c}\text { Yield, \% } \\
y\end{array}$} \\
\hline & $\begin{array}{l}\text { Oleic acid to cetostearyl } \\
\text { alcohol, } \mathrm{mol} / \mathrm{mol}\end{array}$ & $\begin{array}{l}\text { Reaction time, } \\
\text { min }\end{array}$ & $\begin{array}{l}\text { Process temperature, } \\
{ }^{\circ} \mathbf{C}\end{array}$ & & $\mathbf{X}_{1}$ & $X_{2}$ & $\mathbf{X}_{3}$ & \\
\hline 1 & 2 & 3 & 4 & 5 & 6 & 7 & 8 & 9 \\
\hline 1 & 0.9 & 120 & 40 & +1 & -1 & -1 & -1 & 95.05 \\
\hline 2 & 1.1 & 120 & 40 & +1 & +1 & -1 & -1 & 89.29 \\
\hline 3 & 0.9 & 240 & 40 & +1 & -1 & +1 & -1 & 97.42 \\
\hline 4 & 1.1 & 240 & 40 & +1 & +1 & +1 & -1 & 95.17 \\
\hline 5 & 0.9 & 120 & 60 & +1 & -1 & -1 & +1 & 97.56 \\
\hline 6 & 1.1 & 120 & 60 & +1 & +1 & -1 & +1 & 98.07 \\
\hline 7 & 0.9 & 240 & 60 & +1 & -1 & +1 & +1 & 99.13 \\
\hline 8 & 1.1 & 240 & 60 & +1 & +1 & +1 & +1 & 97.66 \\
\hline 9 & 1 & 180 & 50 & +1 & 0 & 0 & 0 & 96.71 \\
\hline 10 & 1 & 180 & 50 & +1 & 0 & 0 & 0 & 97.08 \\
\hline 11 & 1 & 180 & 50 & +1 & 0 & 0 & 0 & 96.37 \\
\hline
\end{tabular}


Based on relevant calculations, the value of the response function was found and regression equations were derived. The relationship of the yield of the desired fraction (Y, \%) and the key modification parameters (ratio of reagents, $\mathrm{mol} / \mathrm{mol}$; reaction time, min; process temperature, ${ }^{\circ} \mathrm{C}$ ) is described by the following equation. In coded variables, it is expressed as follows:

$$
\begin{gathered}
Y=96.16875-1.12125 \mathrm{X}_{1}+1.17625 \mathrm{X}_{2}+1.93625 \mathrm{X}_{3}+ \\
0.88125 \mathrm{X}_{1} \mathrm{X}_{3}-0.88625 \mathrm{X}_{2} \mathrm{X}_{3}-0.68625 \mathrm{X}_{1} \mathrm{X}_{2} \mathrm{X}_{3} .
\end{gathered}
$$

The relationship of the yield of the desired fraction after modification and the key modification parameters in physical variables can be expressed as:

$$
\begin{gathered}
\mathrm{Y}=227.942-158.213 x_{1}-0.479 x_{2}-2.482 x_{3}+2.940 x_{1} x_{3}+ \\
0.572 x_{1} x_{2}+0.010 x_{2} x_{3}-0.011 x_{1} x_{2} x_{3} .
\end{gathered}
$$

Extreme values were found for the relationship (2) as shown in Table $\mathbf{3}$.

Table 3

Extreme values of the regression curve (2)

\begin{tabular}{cccc}
\hline Function value, $\%$ & Ratio of reagents, $\mathbf{~ m o l} / \mathbf{m o l}$ & Reaction time, $\mathbf{m i n}$ & Process temperature, ${ }^{\circ} \mathbf{C}$ \\
\hline Code & $x_{1}$ & $x_{2}$ & $x_{3}$ \\
Yield of the desired fraction & & & 60 \\
Maximum value $y=99.42$ & 0.9 & 240 & 40
\end{tabular}

So, during the process of mathematical treatment, a numerical relationship (expressed as a regression model) of the yield of the desired fraction and the ratio of reagents, reaction time, and process temperature was identified, and rational process parameters were calculated.

Images of the relationship between the yield of the desired product and the quantity of oleic acid in the reaction mixture and esterification time can be found in Fig. 2, 3. Analyzing the above surfaces (the model of the response surface and the projection of the response surface), we can conclude that the maximum yield of the target fraction ( $>98 \%$ ) corresponds to the area with the most intense red color. Thus, carrying out the process with the parameters, corresponding to this zone, will ensure the maximum yield of the fraction (more than $98 \%$ ): the amount of oleic acid (0.88-0.98 mol); process duration (200-260 min).

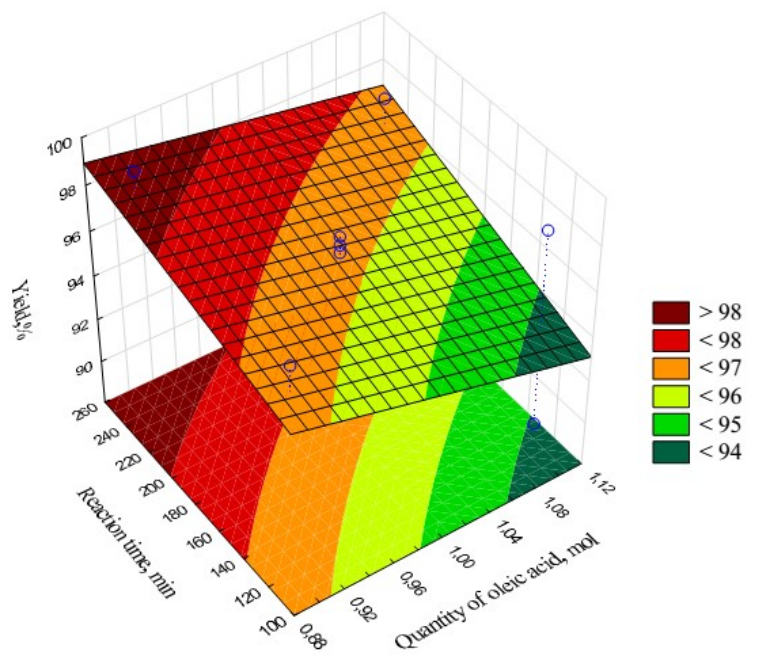

Fig. 2. Yield of the desired product versus the quantity of oleic acid and reaction time: response surface model 


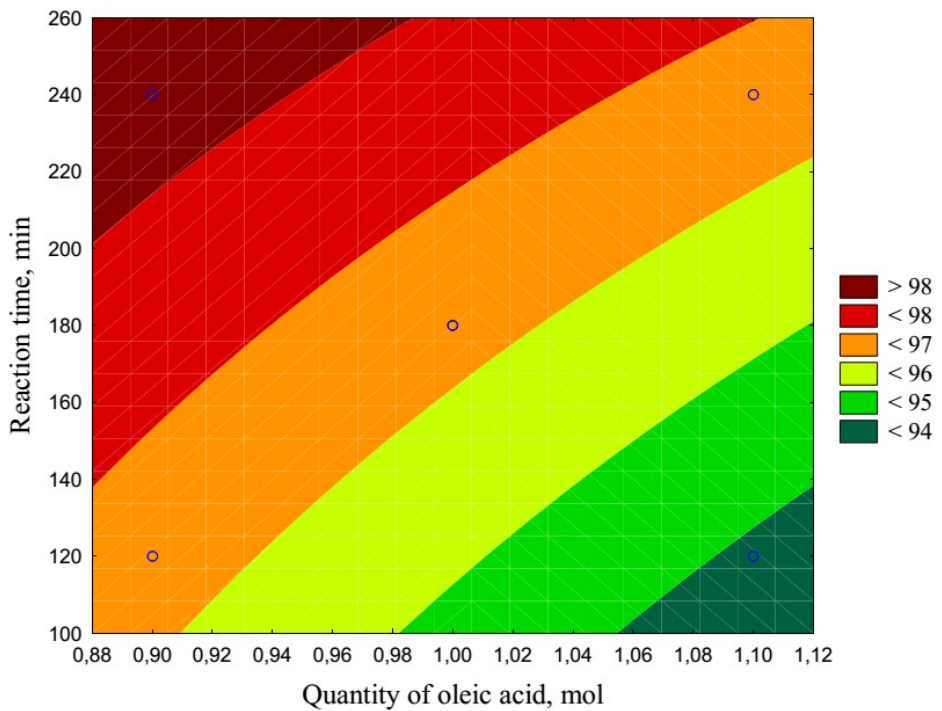

Fig. 3. Yield of the desired product versus the quantity of oleic acid and reaction time: response surface projection

Images of the relationship between the yield of the desired product and the quantity of oleic acid in the reaction mixture and esterification temperature can be found in Fig. 4, 5. Analyzing the above surfaces (the model of the response surface and the projection of the response surface), we can conclude that the maximum yield of the target fraction $(>98 \%)$ corresponds to the area with the most intense red color. Thus, carrying out the process with the parameters, corresponding to this zone, will provide the maximum yield of the fraction (more than $98 \%$ ): the amount of oleic acid $(0.88-1.12 \mathrm{~mol})$; process temperature $\left(54-62^{\circ} \mathrm{C}\right)$.

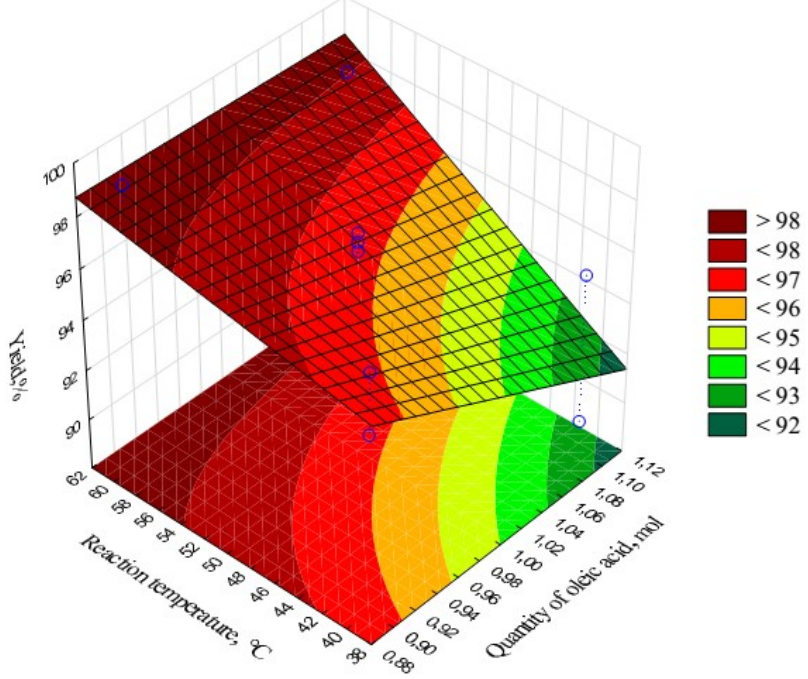

Fig. 4. Yield of the desired product versus the quantity of oleic acid and process temperature: response surface model

Images of the relationship between the yield of the desired product and esterification time and temperature can be found in Fig. 6, 7. Analyzing the above surfaces (the model of the response surface and the projection of the response surface), we can conclude that the maximum yield of the target fraction $(>98 \%$ ) corresponds to the area with the most intense red color. Thus, carrying out the process with the parameters, corresponding to this zone will provide the maximum yield of the fraction (more than $98 \%$ ): the duration of the process (100-260 minutes); process temperature $52-61^{\circ} \mathrm{C}$ ). 
Reports on research projects
(2020), «EUREKA: Life Sciences»

Number 3

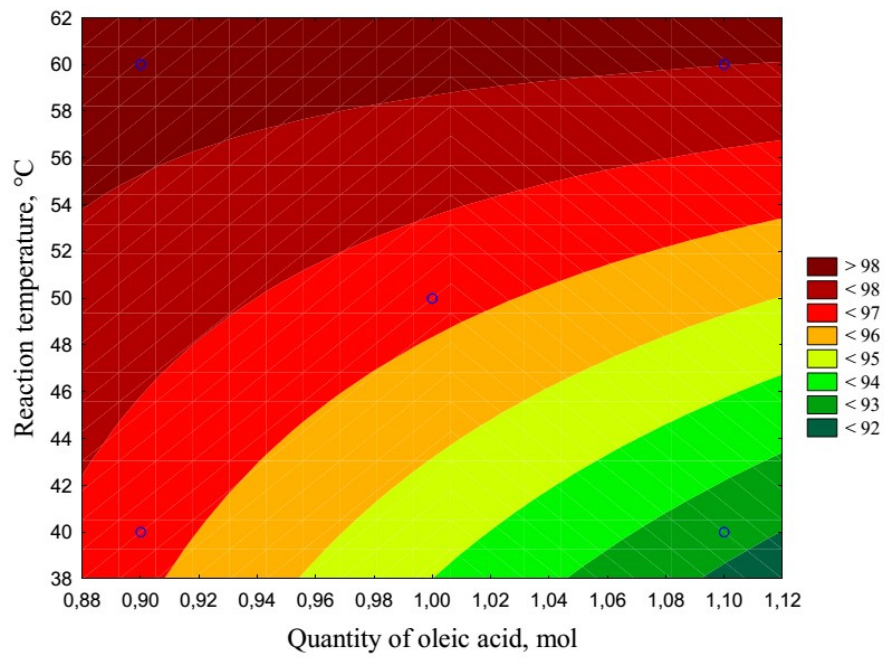

Fig. 5. Yield of the desired product versus the quantity of oleic acid and process temperature: response surface projection

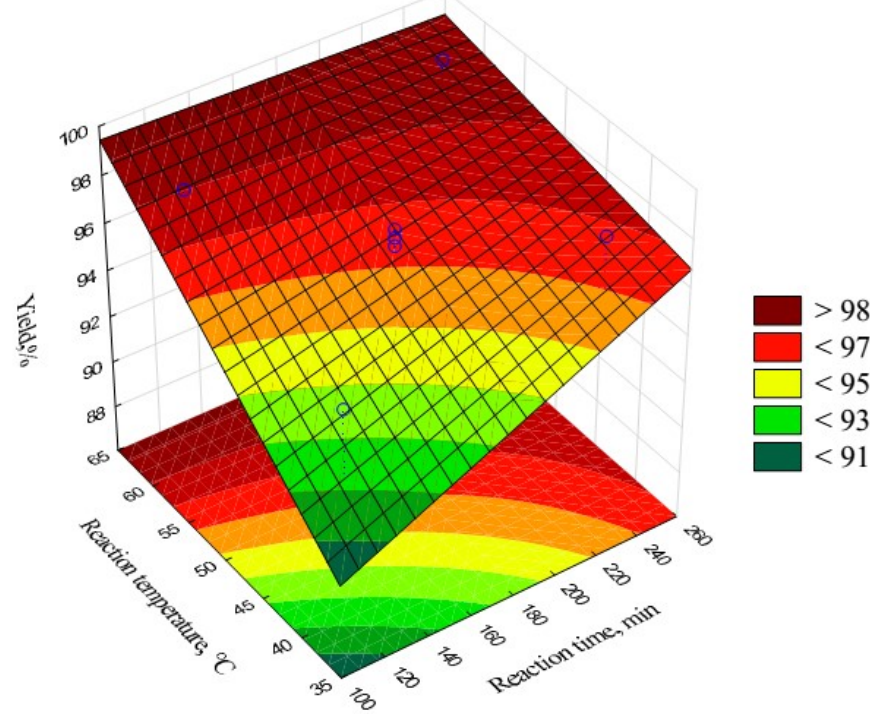

Fig. 6. Yield of the desired product versus the reaction time and process temperature: response surface model

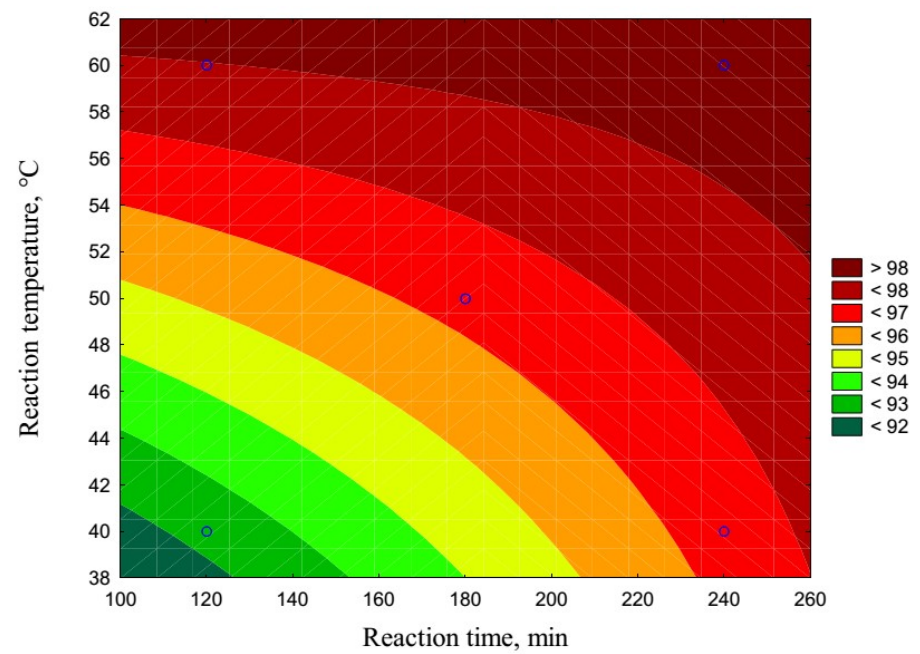

Fig. 7. Yield of the desired product versus the reaction time and process temperature: response surface projection 
Mathematical description (in the form of a regression model) established the rational conditions of the technology of enzymatic esterification of fatty acids with high molecular weight alcohols (Table 3): ratio of reagents $-0.9 \mathrm{~mol} / \mathrm{mol}$, reaction time $-240 \mathrm{~min}$, process temperature $60{ }^{\circ} \mathrm{C}$, which ensures maximum yield of the desired fraction $(99.42 \%)$.

One of the key criteria of quality of modified fats is melting point. The results of studying the melting points of the esters obtained are shown in Table 4.

Table 4

Melting points of ester samples

\begin{tabular}{ccc}
\hline Item No. & Ester & Melting point, ${ }^{\circ} \mathbf{C}$ \\
\hline 1 & Cetyl ester of stearic acid & 56.1 \\
2 & Cetyl ester of oleic acid & 29.6 \\
3 & Cetostearyl ester of stearic acid & 54.55 \\
4 & Cetostearyl ester of oleic acid & 34.65
\end{tabular}

Because there is no national regulatory standard for frying fats, but there is DSTU (Ukrainian Standard) 4335:2004 "Fats for confectionary, cookery, bakery and dairy industry. General specifications", wherein the melting point of cooking fats, and equally frying fats, ranges between 28 and $36{ }^{\circ} \mathrm{C}$. The measurements of melting points in cetyl ester of oleic acid and cetostearyl ester of oleic acid vary from 29.6 to $34.65^{\circ} \mathrm{C}$, which is consistent with the standards. Other samples obtained, namely cetyl ester of stearic acid and cetyl stearyl ester of stearic acid with melting points of $56.1{ }^{\circ} \mathrm{C}$ and $54.55^{\circ} \mathrm{C}$, respectively, are qualified as a component for fatty products.

\section{Conclusions}

Four samples of modified fats were obtained: cetyl ester of oleic acid, cetyl ester of stearic acid, cetostearyl ester of stearic acid, and cetostearyl ester of oleic acid. The use of NaA type zeolites helped increase the yield of esters of fatty acids of high molecular weight alcohols to over $97 \%$. The acid value for cooking oils and fats should not exceed $0.4 \mathrm{mg} \mathrm{KOH} / \mathrm{g}$, therefore after modification the samples were additionally neutralized with a $10 \%$ water/alcohol/glycerin solution of $\mathrm{KOH}$. After that, all of the samples met the specified criterion.

The data underwent mathematical treatment and a numerical relationship (expressed as a regression model) was identified between the yield of the desired product and the ratio of reagents, reaction time, and process temperature, as well as rational process parameters were calculated, i. e. the maximum yield of the desired fraction is achieved at the ratio of reagents of $0.9 \mathrm{~mol} / \mathrm{mol}$, reaction time $240 \mathrm{~min}$, and process temperature $60{ }^{\circ} \mathrm{C}$.

An innovative technology for producing culinary (frying) fats was suggested through esterification of fatty acids with high molecular weight alcohols, using NaA type zeolites, which results in a high-yield alternative to culinary (frying) fats. It is expected to continue research, using other initial materials and chemical catalysts to produce a range of high-quality cooking fats with pre-defined properties.

\section{References}

[1] Rudakova, M. Y., Nikolaeva, Y. V. (2017). Improving the safety of deep-fried foods. Food Industry, 1, 21-26. doi: https:// doi.org/10.7256/2453-8876.2017.1.21205

[2] Samoilov, A. V. (2013). Research of frying fats in the French fries technology. Maslozhirovaya promishlennost, 3, 18-19.

[3] Gallegos-Marin, I., Méndez-Lagunas, L. L., Rodríguez-Ramírez, J., Martinez-Sánchez, C. E. (2020). Influence of osmotic pretreatments on the quality properties of deep-fat fried green plantain. Journal of Food Science and Technology. doi: https:// doi.org/10.1007/s13197-020-04298-5

[4] Yang, J.-H., Park, H.-Y., Kim, Y.-S., Choi, I.-W., Kim, S.-S., Choi, H.-D. (2012). Quality characteristics of vacuum-fried snacks prepared from various sweet potato cultivars. Food Science and Biotechnology, 21 (2), 525-530. doi: https://oi.org/10.1007/ s10068-012-0067-4 
[5] Tutel'yana, V. A., Nechaeva, A. P. (Eds.) (2016). Sovremennye tendentsii razvitiya proizvodstva zhirovyh produktov: nauka, tehnologiya, biznes. Moscow: SPPI, 424.

[6] Hosseini, H., Ghorbani, M., Meshginfar, N., Mahoonak, A. S. (2016). A Review on Frying: Procedure, Fat, Deterioration Progress and Health Hazards. Journal of the American Oil Chemists' Society, 93 (4), 445-466. doi: https://oi.org/10.1007/ s11746-016-2791-z

[7] Pedreschi, F., Moyano, P., Kaack, K., Granby, K. (2005). Color changes and acrylamide formation in fried potato slices. Food Research International, 38 (1), 1-9. doi: https://doi.org/10.1016/j.foodres.2004.07.002

[8] Boskou, G., Salta, F. N., Chiou, A., Troullidou, E., Andrikopoulos, N. K. (2006). Content oftrans,trans-2,4-decadienal in deepfried and pan-fried potatoes. European Journal of Lipid Science and Technology, 108 (2), 109-115. doi: https://doi.org/10.1002/ ejlt.200500236

[9] Moulodi, F., Qajarbeigi, P., Rahmani, K., Haj Hosseini Babaei, A., Mohammadpoorasl, A. (2015). Effect of Fatty Acid Composition on Thermal Stability of Extra Virgin Olive Oil. Journal of Food Quality and Hazards Control, 2 (2), 56-60. Available at: http://jfqhc.ssu.ac.ir/article-1-146-en.pdf

[10] Turan, S., Yalcuk, A. (2013). Regeneration of Used Frying Oil. Journal of the American Oil Chemists' Society, 90 (11), $1761-$ 1771. doi: https://doi.org/10.1007/s11746-013-2325-x

[11] Zinov'ev, A. A. (1952). Himiya zhirov. Moscow: Pishchepromizdat, 551.

[12] Georgiev, D., Bogdanov, B., Hristov, Y., Markovska, I. (2011). Synthesis of NaA Zeolite from Natural Kaolinite. Oxidation Communications, 4, 812-819. 